\title{
Philosophiques
}

\section{Religion et philosophie au Québec et au Canada anglais}

\section{Leslie Armour}

Volume 9, numéro 2, octobre 1982

URI : https://id.erudit.org/iderudit/203198ar

DOI : https://doi.org/10.7202/203198ar

Aller au sommaire du numéro

Éditeur(s)

Société de philosophie du Québec

ISSN

0316-2923 (imprimé)

1492-1391 (numérique)

Découvrir la revue

Citer ce document

Armour, L. (1982). Religion et philosophie au Québec et au Canada anglais.

Philosophiques, 9(2), 307-316. https://doi.org/10.7202/203198ar d'utilisation que vous pouvez consulter en ligne.

https://apropos.erudit.org/fr/usagers/politique-dutilisation/ 


\title{
INTERVENTIONS
}

\section{RELIGION ET PHILOSOPHIE AU QUÉBEC ET AU CANADA ANGLAIS}

\author{
par Leslie Armour
}

De 1665 à 1965, la philosophie au Québec était avant tout une philosophie de religion et également une philosophie religielise. En outre, après 1835 , la philosophie est devenue, pour reprendre les termes de $\mathbf{M}$. Yvan Lamonde «la servante de l'église» ${ }^{1}$.

Toutefois, il n'y avait pas qu'au Québec que l'intérêt pour la religion dominait la philosophie. Northrop Frye a fait remarquer qu'au Canada anglais également, la philosophie était toujours une philosophie religieuse ${ }^{2}$.

Il convient de se demander pourquoi.

La thèse que je propose est celle-ci: d'une part, le Québec et le Canada anglais avaient toujours besoin d'une religion rationnelle et d'autre part, le besoin de philosophie était finalement un besoin de religion rationelle. Pour quelles raisons?

En réalité, les deux peuples étaient composés d'individus qui avaient conservé une certaine idée d'une société organique et communautaire. Ces gens avaient échappé aux influences des philosophes individualistes qui préparaient le terrain pour les révolutions américaine et française. Les français du Québec formaient une société très traditionelle, ainsi que les écossais de l'Ontario et les immigrés américains opposés à la révolution américaine. Ces trois groupes partagaient certaines idées sur la communauté.

1. Yvan Lamonde, La Philosopbie et son enseignement au Québec, (1665-1920). Montréal, Hurtubise $\mathrm{HMH}, 1981$, p. 115. Le présent texte a été rédigé à l'occasion de la parution de ce livre.

2. Frye, Northrop, "Conclusion" in Klinck, Carl, Literary History of Canada, Toronto, University of Toronto Press, 1965 et 1976. La deuxième édition développe les remarques de la première. 
L'artiste et réformiste Paul-Emile Borduas était en exil à Paris quand il écrivit: «j'ai eu la surprise de reconnaître une certaine unité psychique canadienne en découvrant «mes chers ennemis héréditaires» aussi semblables à moi-même que possible» ${ }^{3}$. Les racines sociales sont durables.

Mais existaient-elles, les institutions qui pouvaient soustendre une société communautaire et organique? Au Canada français, seule l'Église survivait à la conquête, face à un pouvoir colonial qui acceptait, en général, les idées «modernes» et individualistes. Au Canada anglais, la situation sociale était même plus critique: il y avait trop d'églises! Le gouvernement colonial - le "family compact» - était surtout anglais, et non pas écossais ou irlandais, et les immigrés américains formaient un groupe qui était souvent plus près que ces derniers du pouvoir colonial. Il existait plusieurs groupes mais le pouvoir manquait. Seule, la tradition chrétienne conservait les fondements d'un concept de communauté. Mais cette tradition était fragmentée. Par conséquent, au Canada anglais, un grand nombre de philosophes cherchaient une doctrine susceptible d'unifier les croyances de la communauté.

Le processus a commencé avec James Beaven, William Lyall, George Paxton Young, John Clark Murray, John Watson; Jacob Gould Schurman et George Blewett et s'est poursuivi jusqu'à la moitié du siècle présent.

Au Québec il n'existait, et il n'existe encore de nos jours, qu'une église importante. Était-il nécessaire de rechercher une raison commune? Souvent, on pense qu'au Québec il était possible de faire sienne une philosophie acceptée par l'église. Mais en réalité, le choix était compliqué et il survenait au Québec des changements qu'on peut mettre en parallèle avec ceux que connaissait le Canada anglais.

Après 1837, il y eu une scission entre les traditionalistes et les ultramontains d'un côté, et les libéraux de l'autre, scission qui correspondait à la division séparant le «family compact» des réformistes au Canada anglais. Il est vrai que l'Église était souvent dominée par un groupe très conservateur et ultramontain, mais

3. Borduas, Paul-Emile, Écrits 1942-1958, Halifax, Nova Scotia College of Art et New York, New York University Press, 1978, p. 153. 
des vagues de fond apportaient toujours un libéralisme ecclésiastique.

En philosophie, une tradition éclectique était très profondément implantée. Publié en 1835, le manuel de philosophie de Jérôme Demers était très célèbre ${ }^{4}$. Les penseurs québécois n'ignoraient pratiquement aucune pensée européenne. Ils connaissaient bien les cartésiens, les empiristes britanniques et les philosophes allemands.

Évidemment, il fallait trouver une philosophie qui puisse servir de base à l'unification du peuple, non seulement à cause de la situation politique chez les gouvernants et gouvernés de l'Église, mais également à cause de la pression exercée par la science sur la doctrine. Il fallait trouver une philosophie pour défendre la foi.

Quelles philosophies pouvaient remplir ce rôle? Elles étaient au nombre de quatre: d'une part la pensée moderne, le mathématisme de Descartes ou l'empirisme de Locke et Hume. De l'autre le «sens commun» des philosophes écossais comme Reid, Stuart et Brown ou les doctrines de Lamennais et de ses amis, élaborées pour résister à cette pensée. Enfin, on trouvait les philosophies catholiques du Moyen Âge, c'est-à-dire la tradition augustinienne et la tradition thomiste.

Chaque pensée avait en réalité ses tenants. On a aujourd'hui tendance à penser que le thomisme s'imposait de lui-même à cause de la pression ecclésiastique de Rome. Mais M. Lamonde, et peut-être surtout $M$. Watzlawik, ont rétabli la vérité en montant que le thomisme était arrivé au Québec plus de vingt ans avant l'encyclique de Léon XIII ${ }^{5}$.

Or, la pensée moderne n'était pas acceptable, pas plus que le cartésianisme ou l'empirisme de Locke et de Hume. La pensée de Descartes avait toujours une coloration très individualiste. Si la philosophie se fonde uniquement sur une expérience intérieure $(J e$ pense donc je suis), il en résulte nécessairement que les principes finaux doivent rester individualistes. Il est vrai que, chez Descar-

4. Demers, Jérôme, Institutiones philosophicae, Québec, Cary et cie., 1835.

5. Lamonde, op. cit. et Watzlawik, Joseph, Leo XIII and the New Scbolasticism, Cebu City, University of San Carlos Press, 1966, pp. 39-71. 
tes, une raison publique a toujours existé, mais c'est bien l'individu qui reconnaît et choisit les principes. De la même façon, les rationalistes, notamment Spinoza et Leibniz, ont élaboré des systèmes fondés sur une certaine idée de la raison publique. Mais chacun d'entre eux avait ses propres principes. Une société communautaire et organique doit pouvoir se fonder sur une expérience publique.

Une telle philosophie existait. Le philosophe français Félicité de Lamennais avait mis sur pied une théorie correspondant dans un sens à la théorie des philosophes écossais tenants du «sens commun». Selon lui, il existait une expérience que l'humanité partageait naturellement. Cette compréhension universelle devait être le fondement de toute spéculation. Or, cette philosophie avait une influence assez importante à Saint-Hyacinthe, le séminaire qui est finalement devenu le coeur du thomisme officiel ${ }^{6}$. Certes, Pierre Thibault a raison: les pères de Saint-Hyacinthe étaient davantage attirés par la politique conservatrice de Lamennais que par son libéralisme théologique ${ }^{7}$.

Les mêmes données du problème existaient au Canada anglais. Les philosophes rejetaient les pensées de Descartes, de Locke et de Hume. Ils recherchaient une expérience publique et adoptaient l'idée du sens commun, prônée par les philosophes écossais. Mais ils n'en restaient pas là. Il est certain que William Lyall, George Paxton Young et John Clark Murray étaient des néophytes de la philosophie du sens commun et que leurs théories étaient fondées sur la conception d'une expérience susceptible d'être partagée par tous les hommes et qui donne le sens d'une réalité extérieure. Mais en fin de compte, Lyall a emprunté quelques éléments à la philosophie augustinienne et Young et Murray sont devenus idéalistes sous l'influence de $\mathrm{Hegel}^{8}$. Pour quelles raisons?

L'expérience philosophique du XIXe siècle engendrait un scepticisme profond quant à la possibilité de l'existence d'une expérience commune. La science, la technologie et les changements sociaux produisaient une transformation radicale de l'expé-

6. Lamonde, op. cit. p. 97

7. Thibault, Pierre Savoir et Pouvoir, Québec, Presses de l'université Laval, 1972.

8. Voir Armour, Leslie et Trott, Elizabeth, The Faces of Reason, An Essay in Pbilosophy and Culture in English Canada, 1850-1950, Waterloo, Sir Wilfred Laurier University Press. 
rience humaine. Or, si l'expérience peut se modifier, peut-on dire qu'il existe vraiment une expérience commune?

Au Québec également, le concept de sens commun était difficile à défendre et ceci pour deux raisons. En premier lieu, tout comme au Canada anglais, de trop grandes divergences d'opinions nuisaient à la crédibilité du concept d'expérience commune. Ensuite, Rome avait condamné Lamennais. Il apparaît évident que l'idée d'un «sens commun» a l'allure d'une vérité déterminée par sondage, mais des raisons plus philosophiques motivaient le rejet de cette théorie par l'Église. Premièrement, la théologie chrétienne exige, selon la conception de l'Église catholique, une dialectique entre l'expérience et la raison. Si l'on ne peut accorder crédit qu'aux expériences des premiers chrétiens, il est impossible d'élaborer une doctrine fondamentale comme celle de la Trinité, non plus que si l'on se fie à la raison pure, comme celle de Leibniz ou de Spinoza. C'est la raison qui se reflète dans l'expérience, dans le processus d'élaboration de telles doctrines. Peut-être y a-t-il une expérience commune au sein de laquelle la raison ne peut pas trouver de structure intelligible, mais l'important est l'expérience intelligible. Lamennais a insisté sur le concept d'une tradition en évolution, mais il a finalement nié la fonction rationnelle de l'église.

Au Canada anglais, après avoir cautionné le sens commun, les philosophes se tournèrent vers Hegel. Mais l'hégélianisme était associé à deux éléments inacceptables au Québec. D'abord, Hegel était protestant; de plus, il pensait que la philosophie pouvait remplacer la religion; ensuite, il défendait une théorie de l'histoire selon laquelle le progrès était inévitable. Au Québec, on pensait que l'histoire de la province était différente de celle des autres. Le Québec n'était pas comme les autres et, par conséquent, son histoire se distinguait de celle des autres ${ }^{9}$. A ce sujet, tous les Québécois étaient unanimes. Par exemple, Jean Charbonneau, condamné par le journal catholique La Vérité ${ }^{10}$, insistait sur le fait que le Québec était le prolongement naturel de la civilisation romaine et que la France s'était écartée du droit chemin ${ }^{1{ }^{1}}$.

\footnotetext{
9. Voir Gagnon, Serge, Québec et ses bistoriens de 1840 à 1920, Québec, Presses de l'université Laval, 1978

10. La Vérité, samedi 31 août 1912.

11. Charbonneau, Jean, Des influences françaises au Canada, Montréal, Beauchemin, 1916-1918
} 
Garneau avait établi l'idée d'un peuple québécois; cette idée de peuple était sans doute empruntée à Michelet ${ }^{12}$. Les philosophes tout comme les historiens avaient accepté cette idée comme fondamentale et les premiers se l'étaient appropriée presque totalement. Mais selon Hegel, la voie du progrès humain était celle qu'avaient suivie la France et l'Allemagne dans les transformations qu'elles avaient connues.

Mais si l'on rejette la thèse d'Hegel, quelles possibilités subsistent? Au nombre des traditions du Moyen Âge, traditions associées à l'Église, on trouvait l'augustinisme et le thomisme. Mais la tradition augustinienne, qui exerçait aux États-Unis une forte influence sur les penseurs catholiques comme Orestes Brownson, tout en ne cessant, par l'intermédiaire de Gioberti, de marquer les catholiques des autres pays, était toujours inacceptable au Québec. En fin de compte, la tendance augustinienne trouvait sa vérité dans la vie intérieure.

«L'ontologisme», doctrine selon laquelle il serait possible de trouver la vérité, véritable substance de Dieu, par l'intermédiaire de la vision intérieure, fut aussi condamnée par l'église, en 1861. Cette condamnation eut un effet limité dans la plupart des pays catholiques, mais au Québec elle fut en général respectée. Il s'agit là, une fois de plus, d'un refus des doctrines individualistes.

Il restait le thomisme qui s'était bien implanté à SaintHyacinthe en 1861, dix-huit ans avant l'encyclique de Léon XIII, publiée en août $1879^{13}$. Au Québec, le thomisme était pratiquement accepté par tous, les autres possibilités ayant évidemment pour les Québécois de graves défauts. Quels avantages présentait le thomisme?

1. D'abord, le thomisme permettait diverses interprétations, parce que cette philosophie contenait non seulement des éléments aristotéliciens mais aussi des éléments néo-platoniciens. On peut souligner à cet égard le monde actuel ou l'au-delà.

2. En second lieu, le thomisme, essentialement démocratique, rendait possible une certaine distance entre l'État et l'Église tout en insistant sur le fait que la séparation n'était pas absolue. $M$.

12. Garneau, François-Xavier, Histoire du Canada depuis sa découverte jusqu'à nos jours, 3 vol., Québec, Aubin et Fréchette, 1845-1848.

13. Voir Lamonde, op. cit. pp. 155-161 et Watzlawik., op. cit pp. 71-75 
Thibault parle donc avec raison d'un contrôle "indirect" de l'Église sur l'État. Mais il existait également d'autres possibilités; cela constitue l'une des raisons pour laquelle la révolution tranquille a été tranquille! ${ }^{14}$.

3. D'autre part, le thomisme prônait la création d'une société communautaire et organique en assurant qu'il existait un bien commun qui primait sur le bien individuel.

4. En outre, au Québec, la révolution industrielle remettait en question la société organique. Le thomisme rendait possible une critique du système capitaliste, mais cautionnait également un refus du socialisme étatiste. Entre les deux existaient le domaine des coopératives, des caisses populaires et des causes sociales défendues par l'Église.

5. Enfin, le thomisme avait été élaboré pour réconcilier science arabe et théologie chrétienne. Il pouvait aussi servir à réconcilier théologie et science, au XIXe siècle.

Au Canada anglais, l'hégélianisme insistait sur la réalité des tensions et d'une pluralité des formes sociales; cette doctrine pouvait également être un encouragement à un pluralisme intellectuel au sein d'une unité qui pouvait conserver les fondements essentiels de la société organique. Les vastes possibilités d'interprétation du thomisme permettaient à cette pensée de remplir la même fonction au Québec.

Cependant, plusieurs différences étaient à noter. Le succès du thomisme au Québec était presque incroyable. En France, il restait une philosophie minoritaire, même parmi les intellectuels catholiques. En Italie, sa popularité était toujours moindre que celle de certaines philosophies idéalistes. En Espagne, les philosophies gardaient un caractère national. Aux États-Unis, le thomisme était finalement devenu une position orthodoxe parmi les intellectuels catholiques, mais il n'avait pratiquement aucune influence en dehors de l'Église.

Comme je l'ai dit, le succès du thomisme au Québec s'explique; toutefois ce succès permettait l'apparition d'un "durcissement» du thomisme. Puisqu'elle ne s'opposait pas à de dures

14. Thibault, op. cit., chapitre VIII 
critiques, cette possibilité semblait aller de soi, notamment parce que l'on avait grand besoin de manuels à l'usage de collèges classiques.

Le manuel le plus populaire était le livre latin en trois volumes de Stanislas Lortie ${ }^{15}$. Mais une tension se faisait toujours sentir. Au niveau universitaire, on trouvait des philosophes comme Louis Lachance et Charles de Koninck qui mettaient en évidence des possibilités très divergentes; toutefois, il y avait toujours une tendance à l'uniformisation.

En outre, d'un point de vue québécois, le thomisme n'était pas véritablement une philosophie à part entière. La notion d'histoire n'y tenait pas une grande place. L'histoire, notamment l'histoire nationale et nationaliste, occupait le centre des préoccupations du Québécois. Naturellement, des philosophes comme Louis Lachance et même Mgr L.A. Paquet ajoutaient au thomisme des éléments d'histoire et de nationalisme.

Mais le besoin de philosophie se trouvait dans la situation sociale et l'accent était toujours mis sur cette philosophie sociale. Pour ce faire, on se servait d'idées religieuses. Pourtant, les philosophes québécois ne faisaient pas tellement de cas des preuves sous-tendant les doctrines les plus fondamentales de la religion. Au Canada anglais, un grand effort était fait pour avancer des preuves de l'existence de Dieu. Au Canada français, il était difficile avant les années soixante de trouver une preuve originale. Les cinq preuves de Saint Thomas suffisaient aux philosophes de l'époque. (Lortie a inclu, dans son ouvrage, la preuve de la conscience que l'on ajoutait normalement aux preuves de Saint Thomas, mais il ne développe pas ce point.)

Au Canada français, on s'intéressait toujours à la philosophie sociale. Le fait qu'on ait remplacé le manuel du Cardinal Zigliara par celui de Lortie révèle que les Québécois voulaient incorporer davantage de politique sociale à leur philosophie, y compris même les concepts de grève et de «lockout». (À ce sujet, il est intéressant de noter que Lortie ne pouvait pas parler de «lockout» sans utiliser le mot anglais ${ }^{16}$.)

15. Lortie, Stanislas, Elementa Pbilosophiae Cbristianae ad Mentem S. Thomae Aquinatis Exposita, 3 vol. 6 édition, Québec, 1929

16. Lortie, op. cit. vol. III, p. 327. En fin de compte, Lortie explique en français le concept de «lockout». Le latin ne suffit pas, il garde le mot "lockout». 
L'ordre thomiste a dominé la philosophie pendant presque cent ans. Mais la révolution était inévitable. Il y avait une crise des institutions et une crise de croyance. Dans la philosophie thomiste, l'Église, en fait toujours l'institution la plus nettement québécoise, unifiait ses vastes opérations. Mais les besoins en matière de services sociaux et d'éducation au sein d'une société industrielle étaient trop compliqués, trop techniques et trop coûteux pour l'Église. Il fallait faire passer certaines fonctions de l'Église à l'État, et une philosophie thomiste de manuel, une philosophie trop rigide, n'avait pas la flexibilité nécessaire pour déterminer les principes de cette opération. Il fallait élaborer une nouvelle philosophie. En fait, les québécois ne cherchaient pas une autre philosophie. En général, ils ne faisaient qu'adopter des modèles empruntés à des sociétés voisines, ce qui ne constituait pas un processus complet. Dans une telle situation, le gouvernement provincial a envisagé une réforme scolaire copiée, encore une fois, sur les modèles américains, dans lesquels Église et État sont séparés.

Le Québec a connu, à l'instar du monde entier, une crise de croyance. C'est une des raisons pour lesquelles le thomisme ne disposait pas, au Québec, des éléments qui lui auraient permis de se défendre efficacement. Il aurait probablement fallu avoir une nouvelle conception de Dieu et de l'homme pour comprendre les nouvelles sciences et pour rendre le christianisme intelligible ${ }^{17}$. Mai la séparation entre foi et raison, grâce à laquelle le thomisme avait relevé les défis du Moyen Âge, était également garante des concepts religieux et protégeait le Québec contre la réforme. Il se pourrait qu'un thomisme moins rigide restât une possibilité importante, mais, d'après moi, dans l'esprit de la plupart des intellectuels québécois, le thomisme est un thomisme de manuel. Personne ne parle du thomisme de Louis Lachance. On garde l'image de celui de Stanislas Lortie.

Il existe encore une société unique québécoise et je pense que l'idée d'une société communautaire reste vivante. D'après moi, également, on trouve, sous différentes formes, dans la vie et dans

17. La philosophie de Pierre Teilhard de Chardin présentait un intérêt pour certains intellectuels québécois qui avaient pris part à la révolution tranquille, tel Jean Le Moyne qui s'intéressait aussi à la pensée de Jacques Maritain. À mon avis, c'est du passé. 
la littérature québécoise, une expérience de la transcendance. Māis comment peut-on l'exprimer?

Quelle pensée pourrait remplacer le thomisme? Il existe des philosophies «existentielles» et "phénoménologiques», mais elles ont une base nettement individualiste. Il est peut-être possible de les utiliser pour exprimer la transcendance, mais pas pour expliquer une société organique.

On trouve aussi les nouveaux «déconstructionistes» français. Ils ont leurs adeptes au Québec. En France, on cherche souvent les moyens de rejeter la tradition et de révolutionner la pensée. Au contraire, au Québec, par le passé, on a la plupart du temps essayé de conserver la tradition et de sauvegarder la civilisation.

La philosophie analytique et le positivisme ont fait des tenants qui ont, actuellement, une certaine influence sur les universités. Mais celle qu'ils exercent sur la vie publique n'est pas grande.

On trouve enfin les diverses philosophies «marxistes» et «marxistes reformées» qui conservent le sens de l'histoire et aussi le sens de la communauté. Mais le marxisme s'oppose toujours au transcendant. Cela constitue, quant à moi, un problème parce que ce monde transcendant reste une importante réalité dans l'expérience québécoise.

Quand on n'a pas de philosophie, il faut emprunter des modèles sociaux à d'autres sociétés. Au Canada anglais, le processus de "dénationalisation» de la philosophie est probablement plus avancé qu'au Québec. L'hégélianisme au Canada anglais n'était pas élaboré avec assez de finesse et les vagues nouvelles de philosophie apportaient des cultures étrangères. De nos jours, il n'est pas facile de définir la tradition anglo-canadienne. Le Québec dispose de plus de ressources grâce au grand nombre de professeurs de philosophie dans les cegeps, et à la foule des étudiants touchés par la philosophie.

Toutefois, on attend l'avènement d'une philosophie vraiment québécoise. En ce qui me concerne, j'attends et j'espère ${ }^{18}$.

Département de philosophie Université d'Ottawa

18. Je voudrais remercier $\mathbf{M}^{\text {me }}$ Dominique Bigras et $\mathbf{M}^{\text {lle }}$ Roselyne Revel de leurs conseils sur le style et sur la grammaire française. Elles ont de beaucoup amélioré mon texte. 